Check for updates

Cite this: RSC Adv., 2018, 8, 856

Received 10th October 2017 Accepted 25th November 2017

DOI: $10.1039 / c 7 r a 11138 j$

rsc.li/rsc-advances

\section{Experimental and theoretical calculation investigation of 2,4-dichlorophenoxyacetic acid adsorption onto core-shell carbon microspheres@layered double hydroxide composites $\uparrow$}

\begin{abstract}
Bo Zhang, ${ }^{a}$ Shiling Yuan, ${ }^{b}$ Dejun Sun, ${ }^{b}$ Yujiang Li (iD *a and Tao $\mathrm{Wu}^{* \mathrm{~b}}$
Layered double hydroxides (LDHs) usually aggregate irregularly and hardly redisperse in water. Moreover, the affinity of LDHs is poor for organic compounds. In this study, three different core-shell composites, i.e. CMS@MgAl-LDH, CMS@NiAl-LDH, and CMS@ZnAl-LDH, were synthesized by direct fabrication of LDH nanoplatelets onto carbon microspheres (CMS) for the removal of the adsorbed 2,4dichlorophenoxyacetic acid (2,4-D). The CMS@LDH composites show good water-dispersity due to the 3D hierarchical sphere structure and high affinity for 2,4-D due to the organic carbon cores that possess abundant hydrophobic compounds. It was found that the adsorption process was rapid, and the time required to reach the sorption equilibrium was within $100 \mathrm{~min}$. The theoretical DFT calculation analysis suggested that the adsorption of 2,4-D on the CMSQLDH composites was dominated by $\pi-\pi$ interactions, ion-exchange, and hydrogen bonding. The core-shell CMSALDH composites can serve as a promising adsorbent that offers a rapid and effective adsorption capacity for the removal of 2,4-D in an aqueous solution.
\end{abstract}

\section{Introduction}

Organic pesticide pollution constitutes one of the most serious environmental problems globally due to the mass production of these pesticides in the industry and their wide usage in agricultural activities. 2,4-Dichlorophenoxyacetic acid (2,4-D), as a representative industrially produced organic pesticide compound, is commonly utilized as a herbicide and plantgrowth regulator in wheat, rice, maize, forest, lawns, and

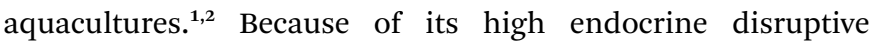
capacity and poor biodegradability, the World Health Organization (WHO) has stipulated that the concentration of 2,4-D in drinking water must be lower than $20 \mu \mathrm{g} \mathrm{L}^{-1}$. $^{2}$ However, 2,4-D tends to leach from soil and rapidly spreads in the surface and groundwater due to the repulsive electrostatic interactions existing between 2,4-D and negative soil sediment. ${ }^{3}$ Hence, it is

${ }^{a}$ Shandong Provincial Key Laboratory of Water Pollution Control and Resource Reuse,
School of Environmental Science and Engineering, Shandong University, Jinan,
250100, PR China. E-mail: yujiang@sdu.edu.cn; Fax: +86-531-88365922; Tel: +86-
531-88365922
${ }^{b}$ Key Laboratory of Colloid and Interface Science of Education Ministry, Shandong
University, Jinan, 250100, PR China. E-mail: wutao@sdu.edu.cn; Fax: +86-531-
88365437; Tel: +86-531-88365437

† Electronic supplementary information (ESI) available. See DOI: $10.1039 / \mathrm{c} 7 \mathrm{ra11138j}$ critical to develop effective approaches to remove 2,4-D from aqueous environments.

Adsorption is a widely used technology to remove organic pollutants from aqueous solutions due to its high efficiency, relatively low-cost, and ease of operation. Many types of adsorbents, such as activated carbon, ${ }^{4}$ graphene oxide, ${ }^{5}$ chitosan, ${ }^{6}$ and biomass materials, ${ }^{7}$ have been used for the removal of 2,4-D. However, these adsorbents possess a number of disadvantages, such as high synthetic cost, low adsorption capacity, and slow adsorption rate, which limit their practical application. Therefore, the design and fabrication of rapid and effective adsorption materials that can overcome the disadvantages of the traditional adsorbents and meet the environmental remediation requirements are highly important.

Layered double hydroxide (LDHs), representing an important class of nanostructured hydrotalcite-like materials, can be described by the general formula $\left[\mathrm{M}^{2+}{ }_{1-x} \mathrm{M}^{3+}{ }_{x}(\mathrm{OH})_{2}\right]^{x+}$ $\left[\left(\mathrm{A}^{m-}\right)_{x / m}\right]^{x-} \cdot n \mathrm{H}_{2} \mathrm{O}$. These layered materials consist of positively hydrotalcite-like host layers that contain M(II) and M(III) metal cations and charge balancing interlayer anions as well as water molecules that can be replaced with other inorganic or organic anions. ${ }^{8-10}$ Due to high structural charge density and ionexchange capability, LDHs possess significant potential application in water treatment. Recently, numerous studies have been focused on the ability of LDHs to remove 2,4-D from aqueous solutions. ${ }^{\mathbf{1 1 1}-13}$ However, the powder LDH adsorbents 
used in these studies usually aggregate irregularly and hardly redisperse in water, ${ }^{\mathbf{1 4}, \mathbf{1 5}}$ which hinders the anion exchange reactions and decreases the adsorption rate. Furthermore, owing to the abundant hydrophilic hydroxyl groups existing in the brucite-like layers, the affinity of LDHs is poor for organic compounds, and they preferentially attract polar water molecules to their surface. The surface property of LDHs can be converted from intrinsic hydrophilic to increasingly hydrophobic by replacing inorganic anions with an anionic surfactant. However, these organic anions are hazardous to the environment if they are exchanged into aqueous solutions. Recently, the development of self-assembly core-shell multifunctional materials has attracted significant interest. The core-shell materials that are fabricated for the tailored functions can offer some novel properties for their use in catalysis, ${ }^{16}$ drug delivery, ${ }^{17}$ gene delivery, ${ }^{18}$ and environmentalrelated applications. ${ }^{\mathbf{1 9 2 0}}$ Moreover, several studies have demonstrated that the fabrication of layered materials with the well-dispersed 3D hierarchical nano- or microstructures, especially the spherical structures, can reduce the aggregation of layered materials and improve their potential for practical applications. ${ }^{15-17,21}$ Therefore, fabrication of a core-shell type $3 \mathrm{D}$ composite via direct assembly of the $\mathrm{LDH}$ nanoplatelets on the surfaces of carbon microspheres (defined as CMS@LDHs) can enhance the dispersibility of the LDHs. Moreover, organic carbon cores that possess abundant hydrophobic compounds can improve the affinity of the synthesized composites for organic pollutants.

The principal objectives of the current study were to synthesize CMS@MgAl-LDH, CMS@NiAl-LDHs and CMS@ZnAl-LDHs composites and investigate the adsorption ability of these composites for the removal of 2,4-D in the aqueous solutions. Then, the mechanism of the interaction between 2,4-D and the CMS@LDH composites during the adsorption process was revealed by the density functional theory (DFT) calculations.

\section{Experimental and theoretical methods}

\subsection{Preparation of the carbon microsphere (CMS) template}

The CMS was synthesized via carbonization of glucose by following a standard hydrothermal method in an aqueous solution. ${ }^{\mathbf{1 6}}$ The detailed synthesis processes have been listed in ESI. $\dagger$

\subsection{Preparation of LDHs}

MgAl-LDHs, ZnAl-LDHs, and NiAl-LDHs were prepared by the co-precipitation method. The detailed procedures are shown in ESI. $\dagger$

\subsection{Direct assembly of LDHs on the surface of CMS}

The CMS@LDH composites were fabricated under the assistance of ultrasonication, the detailed of which is shown in ESI. $\dagger$

\subsection{Characterization}

The morphology and the structure of the CMS and CMS@LDH composites were characterized by scanning electron microscopy (SEM, JSM-6700F, JEOL, Japan). The structures of the samples were identified by a D/Max 2200 PC powder diffractometer (XRD, Riguku, Japan) with $\mathrm{Cu} \mathrm{K} \alpha$ radiation. The changes in the CMS and CMS@LDH composites before and after the adsorption of 2,4-D were investigated via a Fourier transform infrared spectrometer (FT-IR, Perkin-Elmer, U.S.A.) using the KBr pellet technique and an X-ray photoelectron spectroscope (XPS, Kratos, U.S.A.) using a monochromatic $\mathrm{Al} \mathrm{K} \alpha$ source, operating at $150 \mathrm{~W}(10 \mathrm{~mA}, 15 \mathrm{kV})$. The zeta potentials of the CMS and CMS@LDH composites in a $\mathrm{KNO}_{3}$ solution (0.001 M) were determined by a ZetaPALS zeta potential analyzer (Brookhaven, New York, U.S.A.). The specific surface area analysis was conducted using a specific surface area pore size analyzer (JW-BK, 122W, Beijing JWGB Sci. \& Tech. Co., Ltd., China).

\subsection{Adsorption experiments}

The adsorption experiments were performed using a batch equilibrium technique. A 2,4-D stock solution was prepared by dissolving $0.5 \mathrm{~g}$ of 2,4-dichlorophenoxyacetic acid in $1 \mathrm{~L}$ of a $2 \%$ ethanol-in-water solution, followed by dilution to obtain the necessary concentrations. The $\mathrm{pH}$ of the 2,4-D solutions was adjusted to the desired values with $0.1 \mathrm{M} \mathrm{HNO}_{3}$ or $0.1 \mathrm{M} \mathrm{NaOH}$ using a pH meter (PB-10, Sartorius, Germany). The batch adsorption experiments were conducted by mixing $0.05 \mathrm{~g}$ of CMS@LDHs with $50 \mathrm{~mL}$ of the 2,4-D solution with the concentrations ranging from 10 to $200 \mathrm{mg} \mathrm{L}^{-1}$ in a set of $100 \mathrm{~mL}$ Erlenmeyer flasks. The mixing solutions were then shaken using a thermostated shaker (SHZ-82, Jintan, China) at $200 \mathrm{rpm}$ and different temperatures until the adsorption equilibrium was reached. At the end of the adsorption equilibrium period, the mixture was centrifuged at $6000 \mathrm{rpm}$ for $15 \mathrm{~min}$ (LG10-2.4A centrifuge, Jingli, China) and then filtered through $0.45 \mu \mathrm{m}$ filter paper (Pall Life Science). The concentration of 2,4-D in the supernatant was measured using a UV-visible spectrophotometer (1601, Shimadzu, Japan) at $\lambda=283 \mathrm{~nm}$.

\subsection{Computational details}

All the calculations were based on the density functional theory (DFT) method. The calculations regarding the CMS reactions with 2,4-D were performed for non-periodic clusters using a Gaussian 09 program. At the mpwb95/6-31+g(d,p) level, the geometry of CMS, 2,4-D, and 2,4-D-CMS was individually optimized, and then, the adsorption energy was investigated. In addition, the water solvent was also considered during the calculation process. The calculations regarding the reactions of LDHs with 2,4-D were performed using the Dmol3 module in the Material Studio 7.0 software package. The exchangecorrelation function of the generalized gradient approximation GGA-PBE was used. For geometry optimization, the solvation model was considered and the Brillouin zone was sampled with a Monkhorst-Pack grid of $3 \times 3 \times 1 k$-points. The tolerance of the self-consistent field (SCF) convergence was $1 \times 10^{-5}$. The 
adsorption energy, the highest occupied molecular orbital (HOMO)-lowest unoccupied molecular orbital (LUMO) energy gap, and the band gap were also studied.

\section{Results and discussion}

\subsection{Characterization}

The XRD patterns of MgAl-LDHs, NiAl-LDHs, ZnAl-LDHs, and CMS are shown in Fig. S1. $\dagger$ The XRD patterns of CMS@MgAlLDHs, CMS@NiAl-LDHs, and CMS@ZnAl-LDHs obtained before and after the adsorption of 2,4-D are shown in Fig. S2. $\dagger$ It can be seen that all the as-prepared LDH precursors exhibit the sharp and symmetric characteristic diffraction peaks, which indicate that the materials have a well-crystallized hydrotalcitelike phase. ${ }^{8}$ Moreover, the MgAl-LDHs exhibited a better crystallized LDH structure, which could be deduced from its sharper and more-symmetric reflections of (003) and (006) as compared to those of ZnAl-LDHs and NiAl-LDHs. In addition, the XRD pattern of CMS was different from the patterns of LDHs. Only a broad and amorphous-like peak was obtained at $20^{\circ}$, suggesting the amorphous structures of CMS. After coating with LDHs, the obtained CMS@LDH composites exhibited a hydrotalcite structure, but the intensity of the characteristic diffraction peaks decreased, which indicated the random arrangement of the LDH layers on the surface of CMS. After the adsorption of 2,4-D onto the CMS@LDH composites, the XRD patterns of the composites showed a clear difference. On the one hand, the ill-defined and broad peaks of the adsorbed composites, especially CMS@NiAl-LDHs, suggest a poorly crystalline state, which is related to the complexation of 2,4-D with the CMS@LDHs composites. ${ }^{8}$ On the other hand, according to the (003) diffraction peaks, the d-spacing in MgAl-LDHs increased from $7.8 \AA$ to $8.1 \AA$ after the adsorption of 2,4-D. Furthermore, the pattern of the CMS@ZnAl-LDHs had two LDH phases with different interlayer spacings, 0.98 and $0.77 \mathrm{~nm}$, after the adsorption of 2,4-D. These behaviors implied that the 2,4-D molecules were located in the interlayer of LDHs through ion-exchange.

The morphology and microstructures of the CMS, CMS@LDH composites, and LDHs were determined by SEM and TEM. Fig. 1(a) and (b) show that all the as-prepared CMS samples possess a solid spherical morphology and a smooth surface. The measured zeta potential of CMS was $-24.21 \mathrm{mV}$ at a pH of 4.77 (natural pH) (Table S1 $\dagger$ ), which could be attributed to the abundant surface hydroxyl groups. ${ }^{17}$ The TEM images in Fig. S3 $\uparrow$ show that the MgAl-LDHs, NiAl-LDH, and ZnAl-LDHs exhibit finely dispersed platelet-like nanocrystals, and the average particle sizes of MgAl-LDHs, NiAl-LDHs, and ZnAlLDHs are about $100 \mathrm{~nm}, 30 \mathrm{~nm}$, and $500 \mathrm{~nm}$, respectively. As shown in Fig. 1(c)-(h), after being coated with the MgAl-LDHs, NiAl-LDHs, and ZnAl-LDHs nanoplatelets, the entire surface of CMS became rough as compared to the original smooth surfaces. Moreover, the zeta potentials of CMS@MgAl-LDHs, CMS@NiAl-LDHs, and CMS@ZnAl-LDHs were +39.28, +43.19, and $+35.77 \mathrm{mV}$, respectively, at $\mathrm{pH} 4$ (Table S1†), which indicated a successful fabrication of the LDH nanoplatelets on CMS due to the electrostatic attraction. Table S1† show the specific surface areas of the CMS@LDH composites. It can be seen that CMS@ZnAl-LDHs have a smaller surface area than CMS@MgAl-LDHs and CMS@NiAl-LDHs, which can be attributed to the low coverage density of the ZnAl-LDH nanoplatelets on CMS. Moreover, CMS@MgAl-LDHs have the largest surface area due to the better-crystallized structure of MgAlLDHs. ${ }^{22}$ Generally, a large surface area and a high electric charge density could create more active sorption sites. ${ }^{13}$ Thus, CMS@MgAl-LDHs and CMS@NiAl-LDHs may possess a high adsorption capacity for 2,4-D.

The FTIR analysis can be utilized to identify the functional groups and the bonds formed by them. The spectra of the CMS@LDH composites obtained before and after the adsorption 2,4-D are shown in Fig. 2. The FTIR spectra of the MgAlLDHs, NiAl-LDHs, and ZnAl-LDHs are shown in Fig. S4. $\dagger$ The vibration bands at $3467,3472,1626$, and $1631 \mathrm{~cm}^{-1}$ represented the $\mathrm{O}-\mathrm{H}$ stretching vibrations of the hydroxyl groups in the brucite-like layer or from the interlayer water molecules. ${ }^{23}$ The adsorption bands in a low-wavenumber region $\left(400-900 \mathrm{~cm}^{-1}\right)$ were assigned to the $\mathrm{M}-\mathrm{O}$ and $\mathrm{M}-\mathrm{OH}$ lattice vibrations of the cations of the LDH nanoplatelets. ${ }^{24,25}$ For the CMS system (Fig. 2(a)), the bands at $3445 \mathrm{~cm}^{-1}$ were assigned to the vibrations of the $-\mathrm{OH}$ group on the surface of CMS.${ }^{16}$ Additionally, the peaks at $1706 \mathrm{~cm}^{-1}$ and $1615 \mathrm{~cm}^{-1}$ represented the stretching vibrations of $\mathrm{C}=\mathrm{C}$ and $\mathrm{C}=\mathrm{O}$ in CMS, respectively, which were formed by the aromatization of glucose in the hydrothermal treatment process. ${ }^{16}$ After CMS was assembled with LDHs, the bands characteristic of $-\mathrm{OH}, \mathrm{C}=\mathrm{O}$, and $\mathrm{C}=\mathrm{C}$ vibrations could be observed in all samples (Fig. 2A(b)-(d)). In addition, the adsorption bands of $-\mathrm{OH}$ shifted to a lower value in wavenumber as compared to those for LDHs. This redshifting phenomenon could be ascribed to the formation of hydrogen-bonding between the hydroxyl groups on the surface of LDHs and CMS. Moreover, the bands observed at 400$900 \mathrm{~cm}^{-1}$ can be assigned to the $\mathrm{M}-\mathrm{O}$ and $\mathrm{M}-\mathrm{OH}$ lattice vibrations of the cations of the LDH nanoplatelets. ${ }^{24,25}$ All these phenomena imply that the LDH layers are successfully assembled on the CMS surface. The FTIR spectra of all composites after the adsorption 2,4-D are shown in Fig. 2(B). Compared with all clean composites, the composites after the adsorption 2,4-D exhibited some new bands at $1000-2000 \mathrm{~cm}^{-1}$. The peaks near $1481 \mathrm{~cm}^{-1}$ were associated with the $\mathrm{C}=\mathrm{C}$ stretching vibrations of the aromatic ring. The $\mathrm{C}-\mathrm{O}-\mathrm{C}$ asymmetric and symmetric stretching vibrations were observed near 1312 and $1094 \mathrm{~cm}^{-1} \cdot{ }^{13}$ As a result, the characterization and the assignment of these bands proved that 2,4-D was successfully adsorbed on the CMS@LDH composites.

The XPS spectra was used to obtain information about the chemical state of the functional groups existing on the surface of the CMS@LDH composite before and after the adsorption of 2,4-D. As shown in Fig. 3(A), the O 1s peak ( $\sim 532 \mathrm{eV})$ and C 1s peak $(\sim 284.7 \mathrm{eV})$ were obviously observed in CMS. From the high-resolution spectrum of $\mathrm{C}$ 1s for CMS (Fig. S5(A)†), the carbonyl carbon $(\mathrm{C}=\mathrm{O}, \sim 287.9 \mathrm{eV})$, carbon in $\mathrm{C}-\mathrm{O}(\sim 286.2 \mathrm{eV})$, and nonoxygenated carbon $(\mathrm{C}=\mathrm{C}, 284.6 \mathrm{eV})$ can be observed, ${ }^{26,27}$ which is consistent with the results of FTIR. Compared with the survey spectra of CMS obtained before 

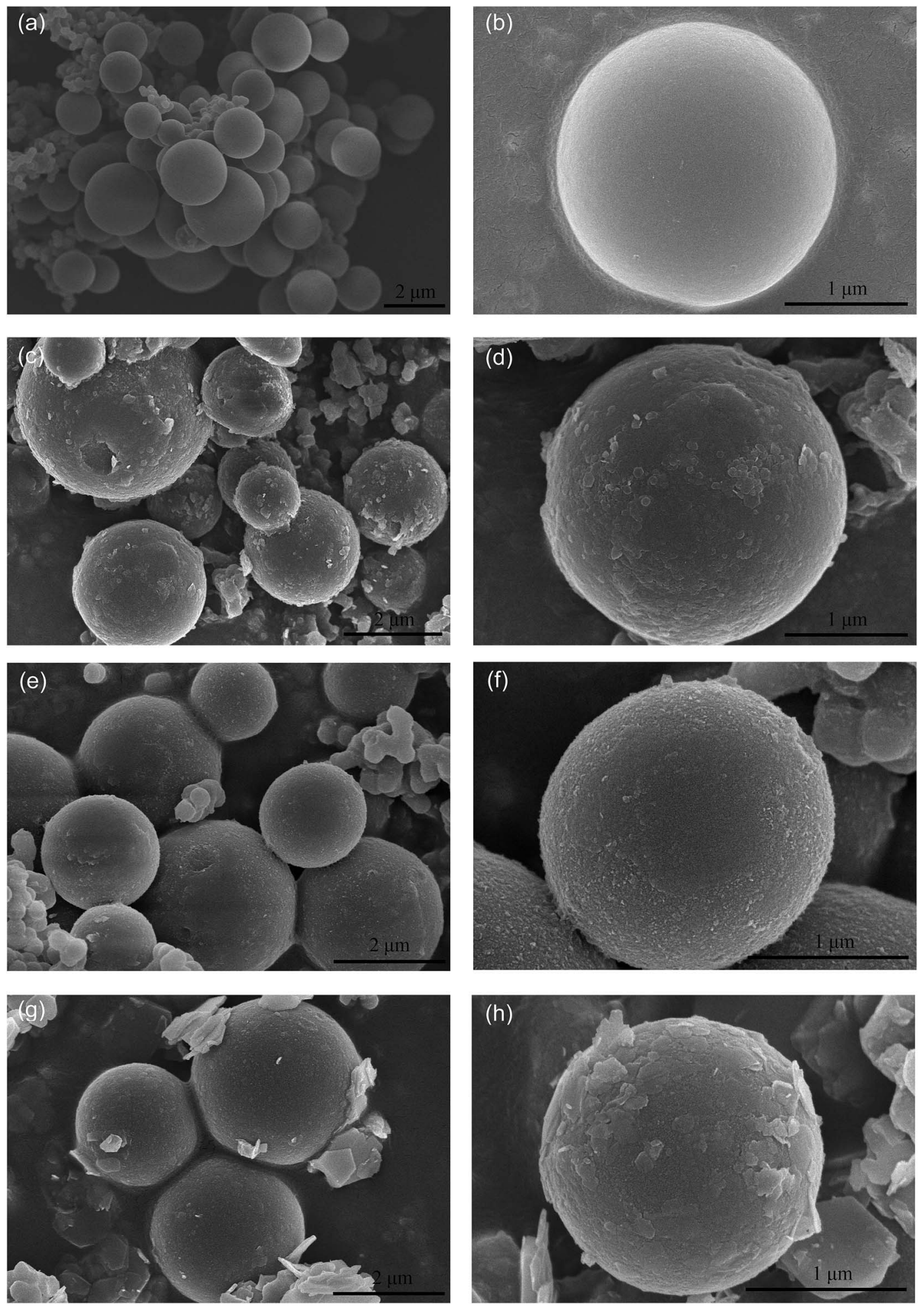

Fig. 1 The SEM image of CMS (a) and (b), CMS@MgAl-LDHs (c) and (d), CMS@NiAl-LDHs (e) and (f), and CMS@ZnAl-LDHs (g) and (h).

assembly with the LDH nanoplatelets, various new peaks, such as $\mathrm{Mg} 1 \mathrm{~s}, \mathrm{Zn} 2 \mathrm{p}$, Ni 2p, Al 2p, and $\mathrm{Cl} 2 \mathrm{p}$, were observed in the spectra of the CMS@LDH composites, which indicated that MgAl-LDHs, NiAl-LDHs, and ZnAl-LDHs were successfully fabricated on the CMS surface. The high-resolution spectra of $\mathrm{C}$ 1s for the CMS@LDH composites are shown in Fig. S5, $†$ and the peak parameters and the contents of the relative functional groups of the CMS and CMS@LDH composites are summarized in Table S2. $\dagger$ After assembly with LDHs, the C 1s peak of composites could be deconvoluted into three overlapped peaks 

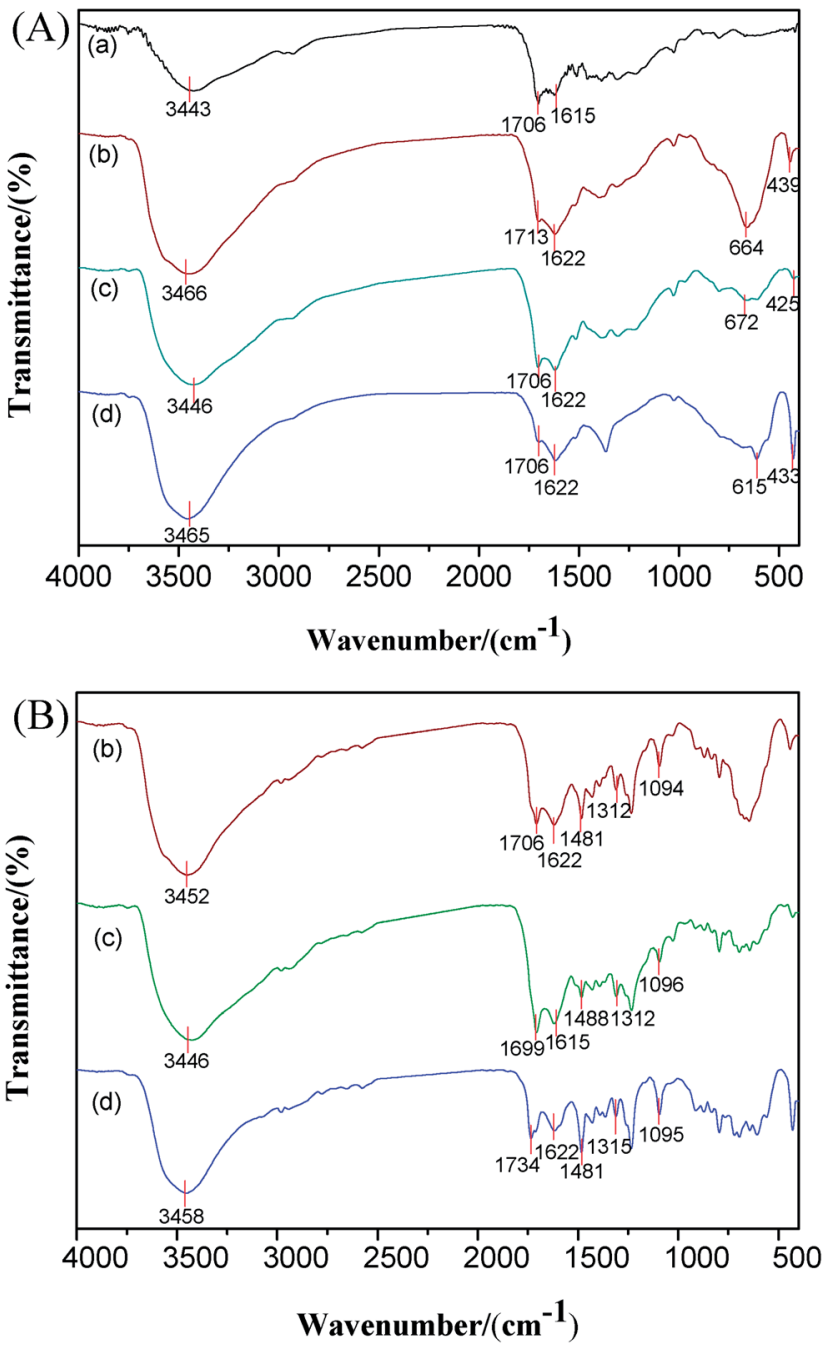

Fig. 2 The FTIR spectra of the samples obtained before the adsorption of 2,4-D (A) and after the adsorption of 2,4-D (B). CMS (a), CMS@MgAl-LDHs (b), CMS@NiAl-LDHs (c), and CMS@ZnAl-LDHs (d)

of $\mathrm{C}=\mathrm{C}, \mathrm{C}-\mathrm{O}$, and $\mathrm{C}=\mathrm{O}$. This means that there are still abundant organic compounds exposed to the surface. As shown in Table $\mathrm{S} 2, \dagger$ the $\mathrm{C}=\mathrm{C}$ peak of the composite before their assembly with LDHs occupied a large part of the total peak, and the content of $\mathrm{C}-\mathrm{O}$ was about $9.86 \%$. After assembly with LDHs, the percentage of $\mathrm{C}-\mathrm{O}$ was markedly increased. This result could be ascribed to the introduction of hydroxyl groups that on the surface of LDHs. Moreover, the binding energy of C-O shifted to a lower value for all composites, which indicated that some interactions between CMS and LDHs occurred. Likely, it is due to the hydrogen bond formed by the oxygen-containing functional groups between CMS and LDHs. The changes in the content of three groups and the alteration of the bonding energy values suggest the assembly of LDHs on the surface of CMS. Compared with the intensities of $\mathrm{C} 1 \mathrm{~s}$ and $\mathrm{Cl} 2 \mathrm{p}$ before the adsorption of 2,4-D, those of $\mathrm{C} 1 \mathrm{~s}$ and $\mathrm{Cl} 2 \mathrm{p}$ were increased after the 2,4-D sorption. The high-resolution spectra of $\mathrm{C} 1 \mathrm{~s}$ for the CMS@LDH composites after the adsorption of 2,4-D are shown in Fig. 3(B-D). After the adsorption of 2,4-D, the spectra of C 1s for the CMS@LDH composites exhibited the peak of carboxylate carbon $(\mathrm{O}-\mathrm{C}=\mathrm{O}, 289 \mathrm{eV})$ that corresponded to the carboxyl groups of 2,4-D. Moreover, as shown in Table S3, $\uparrow$ by comparing the C 1s spectra of the CMS@LDH composites before and after the 2,4-D adsorption, it can be seen that the contents of the $\mathrm{C}=\mathrm{C}$ and $\mathrm{C}=\mathrm{O}$ groups decrease after the adsorption of 2,4-D. However, for $\mathrm{C}-\mathrm{O}$ and $\mathrm{O}-\mathrm{C}=\mathrm{O}$, the contents increased after the adsorption. Based on these results, it is found that 2,4-D can be efficiently adsorbed on the surface of the CMS@LDH composites.

\subsection{Removal performance of the CMS@LDH composites for 2,4-D}

3.2.1. Effect of $\mathbf{p H}$. The adsorption properties of 2,4-D on the CMS@LDH composites as a function of $\mathrm{pH}$ were investigated via batch experiments. As illustrated in Fig. 4, the adsorption of 2,4-D onto three different CMS@LDH composites was greatly dependent on $\mathrm{pH}$. In addition, the removal efficiency decreased with an increase in $\mathrm{pH}$ from 4.0 to 10.0, and the acidic conditions were favorable for the adsorption efficiency. This variation in the removal efficiency in the studied $\mathrm{pH}$ range could be attributed to the surface properties of the CMS@LDH composites and 2,4-D. ${ }^{22}$ The $\mathrm{p} K_{\mathrm{a}}$ of 2,4-D is approximately $2.8,^{2,4,28}$ which indicates that $2,4-\mathrm{D}$ is negatively charged in the $\mathrm{pH}$ range of 4.0-10.0. In addition, as shown in Fig. S6, $\uparrow$ the zeta potentials of three different CMS@LDH composites decreased with an increase in $\mathrm{pH}$ from 4.0 to 10.0 , and the CMS@LDH composites had a high positive zeta potential under the acidic conditions; this indicated that the CMS@LDH composites possessed a good dispersibility in an aqueous solution..$^{29}$ Moreover, the adsorption of 2,4-D onto the CMS@LDH composites could be determined by the electrostatic interaction between them. At lower $\mathrm{pH}$ values, the CMS@LDH composites are protonated and have a higher positive charge density due to the excess of hydrogen ions. Therefore, negatively charged 2,4-D was rapidly adsorbed on the CMS@LDH composites under the effect of electrostatic interaction. However, at higher pH values, the CMS@LDH composites were deprotonated, this reduced the positive charge density. In addition, carbonate was stable, and an excess of $\mathrm{OH}^{-}$ was present in the aqueous media under an alkaline condition..$^{22}$ Hence, the 2,4-D removal efficiency decrease with the increasing $\mathrm{pH}$ was due to the decrease of the electrostatic attraction between 2,4-D and the CMS@LDH composites and the intense competition of $\mathrm{OH}^{-}$, carbonate for the adsorption sites on the CMS@LDH composites.

3.2.2. Effect of contact time. The adsorption capacities of 2,4-D onto the CMS@LDH composites with contact time are shown in Fig. 5. The adsorption amount of 2,4-D increased rapidly with an increase in the contact time for three different composites at the initial adsorption stages and then reached a plateau. The maximum adsorption capacities of 2,4-D onto CMS@MgAl-LDHs, CMS@NiAl-LDHs, and CMS@ZnAl-LDHs were achieved at 60,70 , and $100 \mathrm{~min}$, respectively. The rapid adsorption rate was mainly ascribed to the novel properties of the self-assembly core-shell composites. When the LDH 

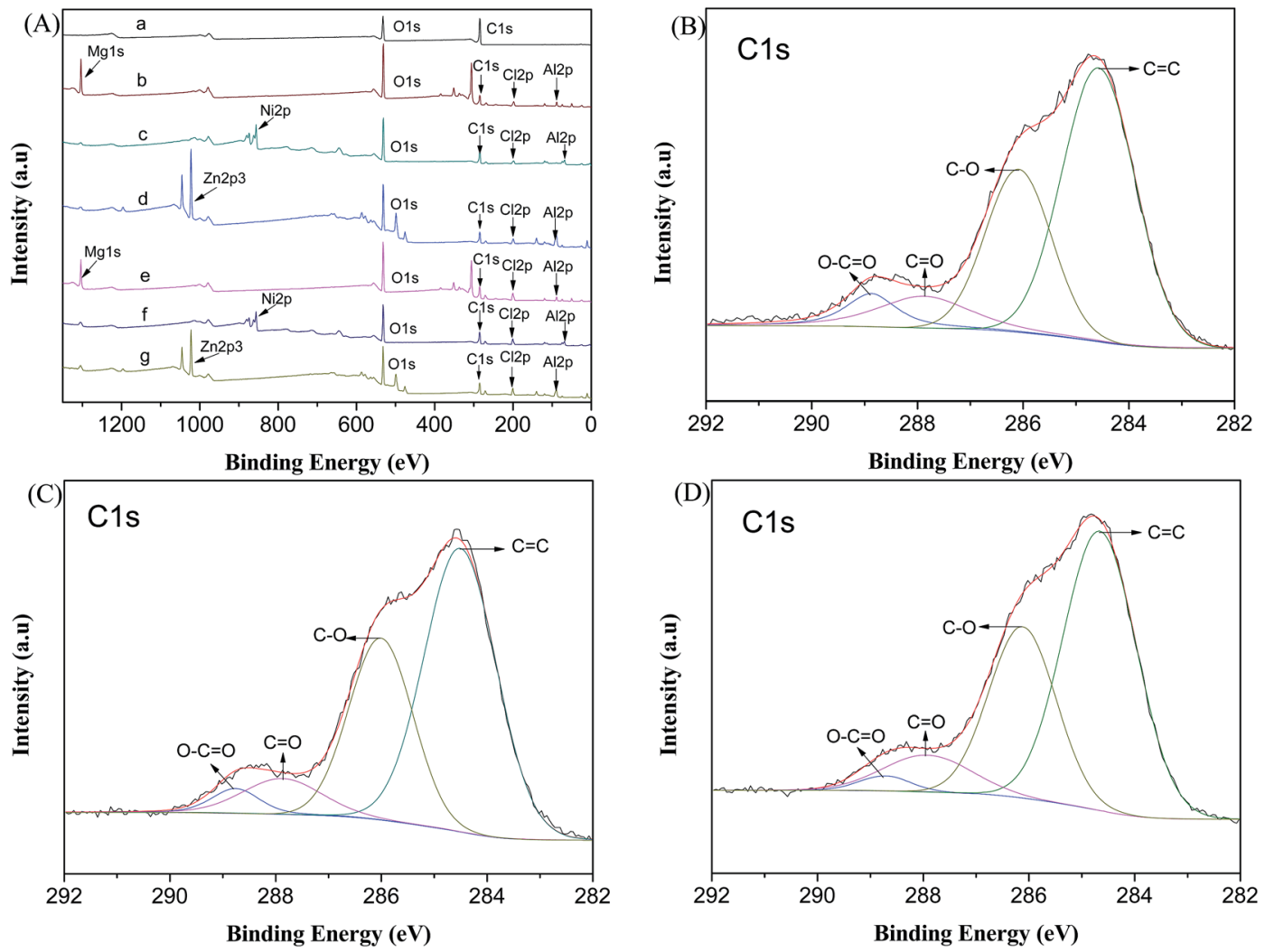

Fig. 3 The survey XPS spectra (A) of CMS (a), CMS@MgAl-LDHs (b), CMS@NiAl-LDHs (c), and CMS@ZnAl-LDHs (d) before the adsorption of 2,4$\mathrm{D}$ and CMS@MgAl-LDHs (e), CMS@NiAl-LDHs (f) and CMS@ZnAl-LDHs (g) after the adsorption of 2,4-D; the HR-XPS spectra of C 1s for CMS@MgAl-LDHs (B), CMS@NiAl-LDHs (C), and CMS@ZnAl-LDHs (D) after the adsorption of 2,4-D.

nanoplatelets were assembled on CMS to form the CMS@LDH composites, the dispersibility of LDHs improved; this reduced the diffusion resistance between LDHs and 2,4-D. Furthermore, the organic CMS core could improve the affinity of the composites for 2,4-D due to the abundant hydrophobic compounds exposed to the surface of the core-shell composites. Therefore, the adsorption rate was improved due to these features. Numerous studies have investigated the adsorption

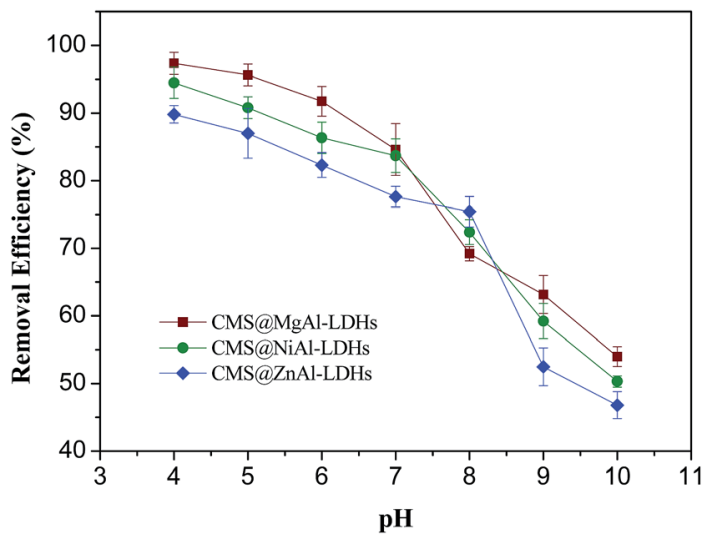

Fig. 4 The effect of the initial $\mathrm{pH}$ on the removal efficiency of the CMS@LDH composites for 2,4-D (initial 2,4-D concentration = $100 \mathrm{mg} \mathrm{L}^{-1}$, absorbent dosage $=1 \mathrm{~g} \mathrm{~L}^{-1}$, contact time $=12 \mathrm{~h}$ at temperature $298 \mathrm{~K}$ ). capacity of 2,4-D onto LDHs. As shown in Table S4, $\uparrow$ MgAlLDHs and NiAl-LDHs containing chloride ion could reach an equilibrium within $6 \mathrm{~h}$ at the initial 2,4-D concentration of $200 \mathrm{mg} \mathrm{L}^{-1}$. Although ZnAl-LDHs are stable and durable in an aqueous solution, ${ }^{30}$ the adsorption needs $12 \mathrm{~h}$ in the concentration range of $221-884 \mathrm{mg} \mathrm{L}^{-1}{ }^{12}$ Calcined hydrotalcite was effective to remove anionic pollutants through the memory

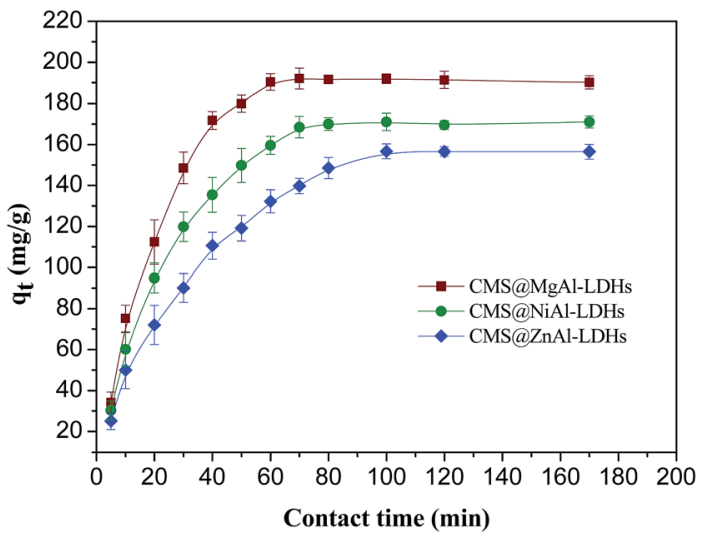

Fig. 5 The effect of the contact time on the adsorption capacities of the CMS@LDH composites for 2,4-D (initial 2,4-D concentration = $200 \mathrm{mg} \mathrm{L}^{-1}$, adsorbents dosage $=1 \mathrm{~g} \mathrm{~L}^{-1}, \mathrm{pH}=4.0$ at temperature 298 K). 
effect and needed $24 \mathrm{~h}$ to reach an equilibrium at the initial 2,4-D concentration of $221 \mathrm{mg} \mathrm{L}^{-1}$ and an adsorbent dosage of $1.5 \mathrm{~g} \mathrm{~L}^{-1} .{ }^{13}$ When the adsorbent dosage increased to $4 \mathrm{~g} \mathrm{~L}^{-1}$, the adsorption rate was improved due to the abundant adsorption site, but the adsorption process still required $6 \mathrm{~h}$ to reach the equilibrium. ${ }^{\mathbf{1 1}}$ In addition, although the calcined $\mathrm{Zn}-\mathrm{Al}-\mathrm{Zr}$-LDHs had a higher charge density and a smaller particle size, ${ }^{1}$ the adsorption required $1-5 \mathrm{~h}$ at a concentration of $100 \mathrm{mg} \mathrm{L}^{-1}$. The long adsorption time could be attributed to the aggregation of the LDH nanosheets and the highly hydrophilic surface properties of LDHs. As a result, the diffusion of the 2,4-D species from the aqueous solution to the internal surface could be hindered, thus, the adsorption rate was slow.

3.2.3. Adsorption isotherms. The adsorption isotherm constitutes a basic measurement to detect the adsorption behavior. To elucidate the detailed interactions of the adsorption of 2,4-D onto CMS@LDHs, the Langmuir ${ }^{31}$ and Freundlich $^{32}$ isotherms were used to describe the relationship between the amount of the 2,4-D adsorbed and its equilibrium concentration in the aqueous solution at a constant $\mathrm{pH}$ and temperature. ${ }^{33}$ The adsorption isotherm of the CMS@LDH composites at $298 \mathrm{~K}$ and those of the CMS@MgAl-LDH, CMS@NiAl-LDH, and CMS@ZnAl-LDH composites at all temperatures $(298 \mathrm{~K}$, $308 \mathrm{~K}$, and $318 \mathrm{~K}$ ) are shown in Fig. 6. The nonlinear form of the Langmuir and Freundlich isotherm equations is calculated as follows:

$$
\begin{aligned}
& q_{\mathrm{e}}=\frac{q_{\mathrm{m}} K_{\mathrm{L}} C_{\mathrm{e}}}{1+K_{\mathrm{L}} C_{\mathrm{e}}} \\
& q_{\mathrm{e}}=K_{\mathrm{F}} C_{\mathrm{e}}{ }^{1 / n}
\end{aligned}
$$

where $q_{\mathrm{e}}\left(\mathrm{mg} \mathrm{g}^{-1}\right)$ is the amount of 2,4-D adsorbed onto the CMS@LDHs, $q_{\mathrm{m}}\left(\mathrm{mg} \mathrm{g}^{-1}\right)$ is the theoretical maximum adsorption capacity, $C_{\mathrm{e}}\left(\mathrm{mg} \mathrm{L}^{-1}\right)$ is the equilibrium solution concentration, $K_{\mathrm{L}}$ is the Langmuir constant related to the adsorption energy and the affinity of binding sites, ${ }^{31,33}$ and $K_{\mathrm{F}}$ and $n$ are the Freundlich constants that are related to the capacity and the intensity of the adsorption, respectively. ${ }^{32}$

The fitted values of the parameters for two isotherms based on the experimental data are shown in Table 1 . All $R^{2}$ values at the studied temperatures obtained from the Langmuir isotherm were higher than those obtained for the Freundlich isotherm, which indicated that the Langmuir isotherm fitted the experimental data better than the Freundlich isotherm at the studied temperatures. Therefore, the adsorption of 2,4-D onto CMS@LDHs was homogeneous and monolayer sorption. The maximum adsorption capacities of CMS@MgAl-LDHs, CMS@NiAl-LDHs, and CMS@ZnAl-LDHs at $298 \mathrm{~K}$ were 287.50, 265.99 , and $223.02 \mathrm{mg} \mathrm{g}^{-1}$, respectively, which indicated that CMS@MgAl-LDHs and CMS@NiAl-LDHs possessed superior adsorption capacities. This behavior could be ascribed to two aspects: one is that CMS@MgAl-LDHs and CMS@NiAl-LDHs have higher surface areas, which provide more active sorption
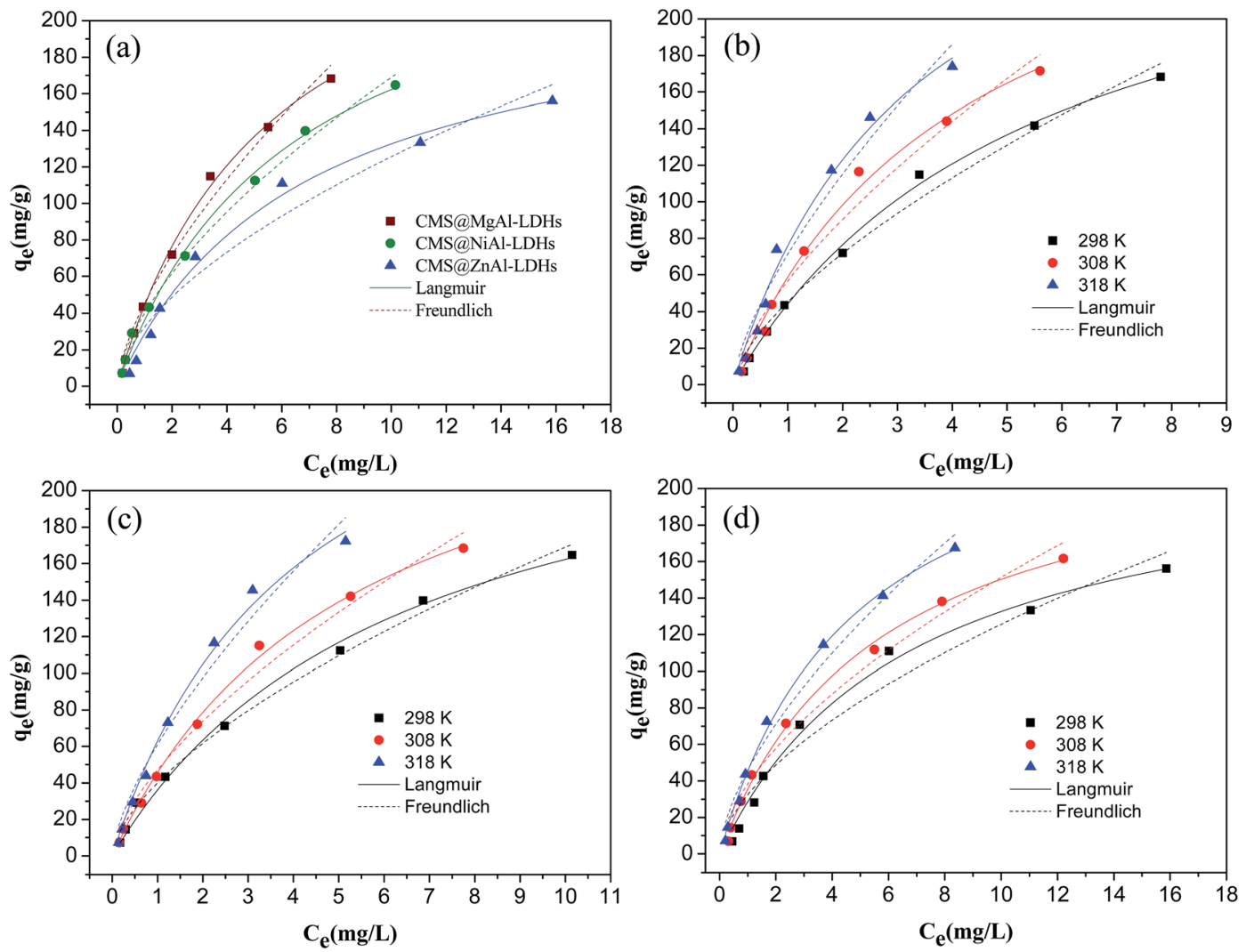

Fig. 6 The adsorption isotherms of 2,4-D onto the CMS@LDHs composites at $298 \mathrm{~K}$ (a) and CMS@MgAl-LDHs (b), CMS@NiAl-LDHs (c), and CMSaZnAl-LDHs (d) at different temperatures (adsorbent dosage $=1 \mathrm{~g} \mathrm{~L}^{-1}, \mathrm{pH}=4.0$, contact time $=24 \mathrm{~h}$ ). 
Table 1 Langmuir and Freundlich isotherm parameters for the adsorption of 2,4-D onto the CMS@LDH composites

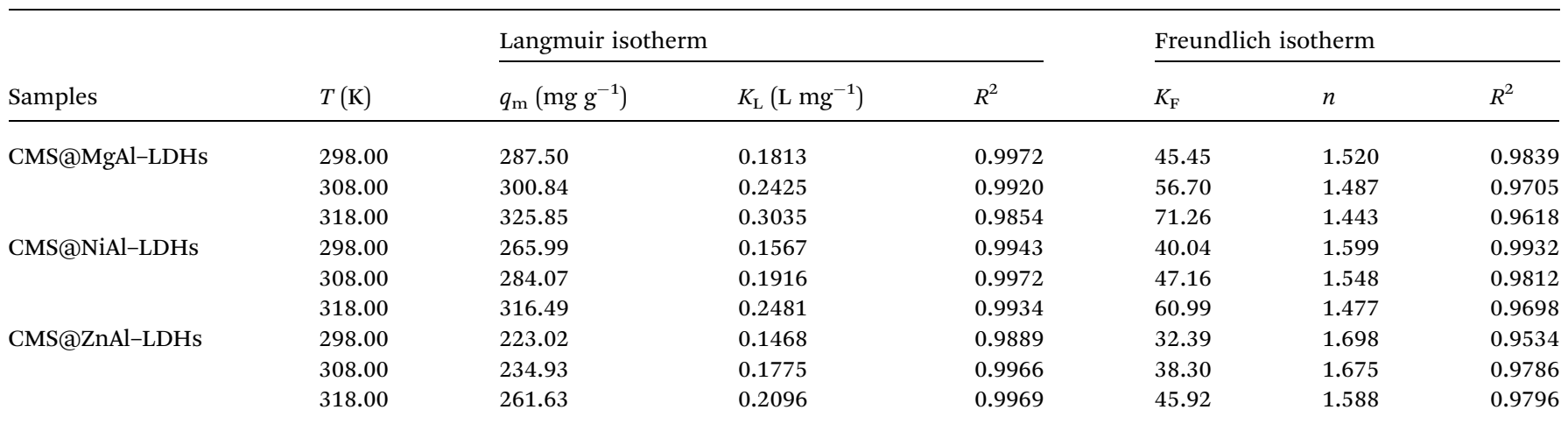

sites for the 2,4-D adsorption onto their surface. The other is that the zeta potentials of CMS@MgAl-LDHs and CMS@NiAlLDHs were higher than that of CMS@ZnAl-LDHs. As abovementioned, the negative 2,4-D ions were adsorbed by the positive CMS@LDHs due to electrostatic attraction. Therefore, the high zeta potential could produce more charge density, which made the driving force between 2,4-D and the CMS@LDH composites stronger. Regarding the coefficients of the Freundlich model, the values of $K_{\mathrm{F}}$ for three composites increased with the increasing temperature, which illustrated that the adsorption capacities of three composites were increased with the increasing temperature.

3.2.4. Thermodynamic parameters. To obtain a better understanding of the nature of the adsorption process and the potential applications of the adsorbent, thermodynamic parameters, including Gibbs free energy $\left(\Delta G^{0}\right)$, enthalpy $\left(\Delta H^{0}\right)$, and entropy $\left(\Delta S^{0}\right)$, were obtained from the following equations: $^{34}$

$$
\begin{aligned}
& \Delta G^{0}=-R T \ln K_{\mathrm{L}} \\
& \ln K_{\mathrm{L}}=\frac{\Delta S^{0}}{R}-\frac{\Delta H^{0}}{R T}
\end{aligned}
$$

where $K_{\mathrm{L}}$ represents the Langmuir constants, $R$ is the universal gas constant $\left(8.314 \mathrm{~J} \mathrm{~mol}^{-1} \mathrm{~K}^{-1}\right)$, and $T$ is the temperature $(\mathrm{K})$. $\Delta H^{0}$ and $\Delta S^{0}$ were calculated from the plot and intercept of van't Hoff plots of $\ln K_{\mathrm{L}}$ versus $1 / T$.

All the thermodynamic parameters for the adsorption of 2,4-D are shown in Table S5. $\dagger$ The negative $\Delta G^{0}$ values revealed that the adsorption process was spontaneous and thermodynamically feasible under the experimental conditions. The values of $\Delta G^{0}$ decreased from -9.79 to $-12.15 \mathrm{~kJ} \mathrm{~mol}^{-1},-9.24$ to $-11.54 \mathrm{~kJ} \mathrm{~mol}^{-1}$, and -8.64 to $-10.59 \mathrm{~kJ} \mathrm{~mol}^{-1}$ for CMS@MgAl-LDHs, CMS@NiAl-LDHs and CMS@ZnAl-LDHs, respectively, in the temperature range of 298-318 K, which suggested that the adsorption was more favorable at high temperatures. The enthalpy change $\Delta H^{0}$ was positive for the adsorption of 2,4-D onto the CMS@LDH composites, which indicated that the adsorption process was endothermic. This behavior can be attributed to ion-exchange occurring between $\mathrm{Cl}^{-}$from the interlayer of LDHs and 2,4-D. ${ }^{22}$ As previously mentioned in Section 3.1, when 2,4-D was adsorbed on the CMS@LDH composites, the crystallinity of the CMS@LDH composites decreased and further increased the disorder of the solid/liquid interface, resulting in a positive value of $\Delta S^{0}$.

\subsection{Density functional theory (DFT) calculations}

To investigate the adsorption mechanism of 2,4-D onto the CMS@LDH composites from a microscale perspective, the

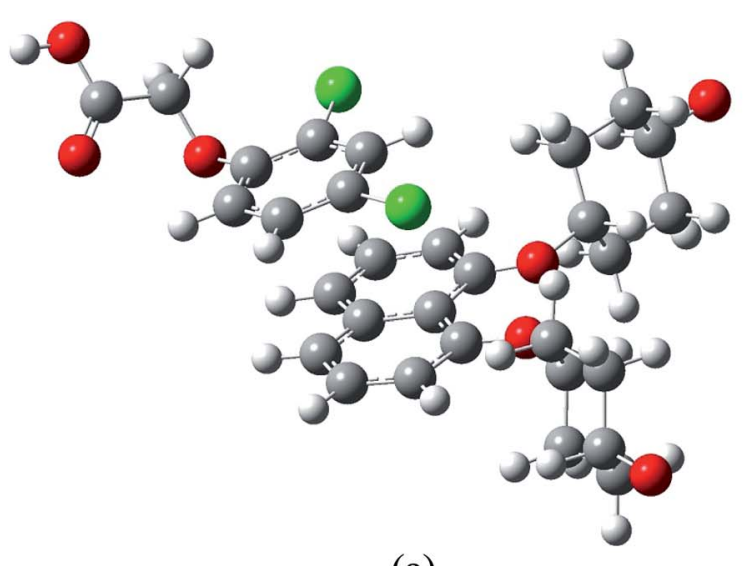

(a)

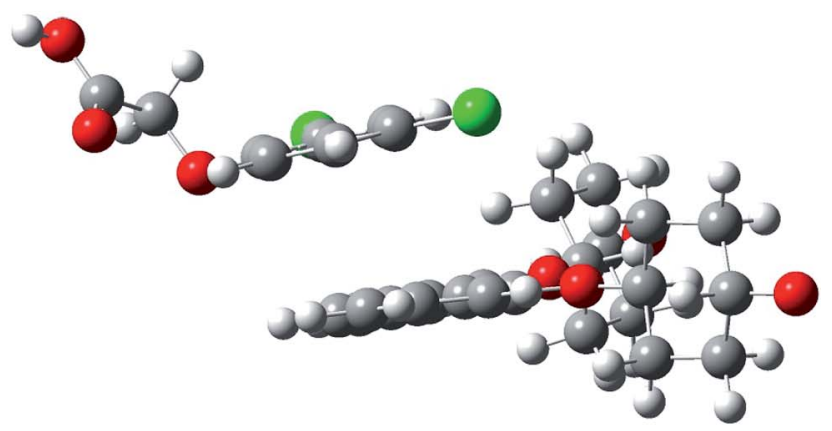

(b)

Fig. 7 The optimized geometrical structure of 2,4-dichlorophenoxyacetic acid adsorbed onto $-\left[\mathrm{C}_{22} \mathrm{H}_{26} \mathrm{O}_{4}\right]$ - depicting the side view ( $\mathrm{a}$ and $\mathrm{b}$ ). Red atoms are oxygen, white atoms are hydrogen, dark green atoms are chlorine, and gray atoms are carbon. 
interaction between 2,4-D and the CMS@LDH composites was modeled by the DFT calculations. As shown in Fig. S7, $\uparrow$ $-\left[\mathrm{C}_{22} \mathrm{H}_{26} \mathrm{O}_{4}\right]$ - was selected as a key functional group for the 2,4$\mathrm{D}$ adsorption due to the fact that CMS consisted of aromatic compounds and oligosaccharides. ${ }^{\mathbf{1 6}}$ Fig. 7 illustrates the geometries of 2,4-D adsorbed onto $-\left[\mathrm{C}_{22} \mathrm{H}_{26} \mathrm{O}_{4}\right]-$. The binding energy was calculated to be $-10.76 \mathrm{kcal} \mathrm{mol}^{-1}$ for $2,4-\mathrm{D}$, indicating that 2,4-D could be adsorbed by $-\left[\mathrm{C}_{22} \mathrm{H}_{26} \mathrm{O}_{4}\right]-$ through a strong interaction. Moreover, after being adsorbed onto $-\left[\mathrm{C}_{22} \mathrm{H}_{26} \mathrm{O}_{4}\right]-$, the rings of 2,4-D were parallel to those of $-\left[\mathrm{C}_{22} \mathrm{H}_{26} \mathrm{O}_{4}\right]-$. This behavior could be caused by the strong $\pi-\pi$ interactions between 2,4-D and $-\left[\mathrm{C}_{22} \mathrm{H}_{26} \mathrm{O}_{4}\right]-.{ }^{35}$ Consequently, it was the strong interaction between 2,4-D and CMS that enhanced the affinity of the CMS@LDH composites for 2,4-D during the adsorption process and improved the adsorption rate.

In addition, we focused on studying the interaction between 2,4-D and LDHs and selected MgAl-LDHs as an example. Fig. S8 $\uparrow$ describes the optimized structure of the MgAl-LDH layers, and the optimized geometries of 2,4-D and $\mathrm{Cl}$ ions adsorbed onto the LDH layers are displayed in Fig. 8. The adsorption energy $\left(E_{\text {ad }}\right)$ was calculated as follows:

$$
E_{\mathrm{ad}}=E_{\mathrm{LDHs}+2,4-\mathrm{D} \text { or } \mathrm{Cl}}-E_{\mathrm{LDHs}}-E_{2,4-\mathrm{D} \text { or } \mathrm{Cl}}
$$

where $E_{\mathrm{LDHs}+2,4-\mathrm{D} \text { or Cl }}$ is the energy of the combined LDH and 2,4-D ion system for LDHs and $\mathrm{Cl}$ ions and $E_{\mathrm{LDHs}}$ and $E_{2,4-\mathrm{D} \text { or Cl }}$ correspond to the energy of the isolated LDHs and 2,4-D or $\mathrm{Cl}$ ions, respectively. The value of $E_{\text {ad }}$ provides information about the nature of the adsorption process and can also be used to show the magnitude of the interaction between the adsorbent and the targets. For example, a positive value of $E_{\text {ad }}$ indicates that the adsorption process is endothermic and the reaction system is unstable, whereas a negative $E_{\text {ad }}$ means that the adsorption process is exothermic and the reaction system is stable. Moreover, a smaller $E_{\text {ad }}$ shows a stronger interaction and a more stable reaction system. As shown in Table 2, the $E_{\text {ad }}$ values were calculated to be -3.16 and $-3.99 \mathrm{eV}$ for $\mathrm{Cl}$ and 2,4-D ions, respectively, showing that $\mathrm{Cl}$ and 2,4-D ions could be adsorbed by the LDH layers through strong interactions, and the main interaction mechanism was chemisorption. ${ }^{27,36}$ However, a smaller $E_{\text {ad }}$ of 2,4-D and the LDH layers suggests the stronger interactions between them, and the 2,4-D-composite system is more stable than the Cl-composite system. Moreover, the $E_{\text {ad }}$ between 2,4-D and the LDH layers is much larger

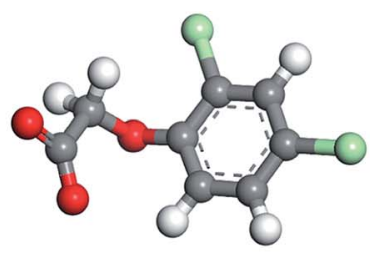

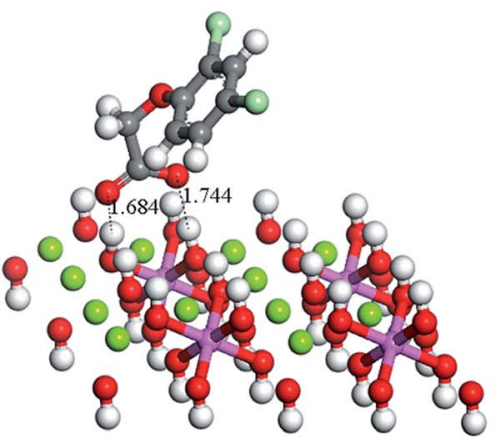

(a)

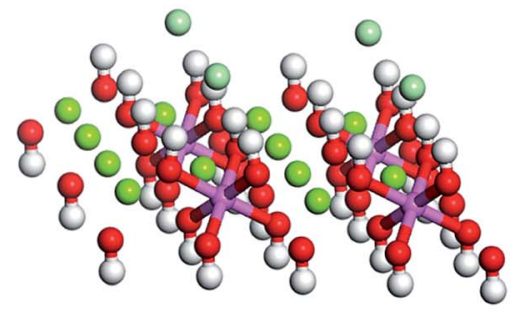

(b)

Fig. 8 2,4-Dichlorophenoxyacetic acid alone and 2,4-dichlorophenoxyacetic acid adsorbed onto the $4 \times 4$ LDH nanosheets depicting the side view (a). Chloride ion alone and chloride ion absorbed onto the $4 \times 4 \mathrm{LDH}$ nanosheets depicting the side view (b).

Table 2 Adsorption energy (eV), HOMO-LUMO energy gap, and band gap values for $\mathrm{Cl}$, 2,4-D ions, and Cl, 2,4-D ions adsorbed on LDHs

\begin{tabular}{llllll}
\hline System & $E_{\text {ad }}(\mathrm{eV})$ & HOMO & LUMO & HOMO-LUMO gap & Band gap (eV) \\
\hline Cl & - & -0.1796 & 0.4515 & 0.6311 & - \\
LDHs-Cl & -3.1565 & - & - & -0.0605 & 0.1231 \\
$2,4-D$ & - & -0.1836 & - & - & 4.868 \\
LDHs-2,4-D & -3.9937 & - & & 4.918
\end{tabular}


than the binding energy between 2,4-D and CMS, which indicates that the adsorption process is mainly dominated by LDHs. The HOMO-LUMO energy gap and the band gap can determine the reactivity of the reaction system, and a smaller HOMOLUMO energy gap or band gap suggests a high reactivity and an unstable system. ${ }^{37}$ As listed in Table 2, the reactivity of the 2,4-D ions was higher than that of the $\mathrm{Cl}$ ions because of the smaller HOMO-LUMO energy gap. However, after 2,4-D and $\mathrm{Cl}$ ions were adsorbed on the LDH layers, the band gap of the 2,4-D composite system was higher than that of the Cl-composite system, which further confirmed that the 2,4-D-composite system had a higher stability and there were stronger interactions between the 2,4-D ions and the LDH layers. These results validate that when the 2,4-D and $\mathrm{Cl}$ ions exist simultaneously, the LDH layers would preferentially adsorb 2,4-D ions, which means that the $\mathrm{Cl}$ ions existing on the surface or in the interlayer of LDHs can exchange with 2,4-D ions. Fig. S9† shows the most stable adsorption structure of 2,4-D adsorbed onto the LDH layers. When the 2,4-D molecules were adsorbed onto the LDH layers, the hydrogen bonds between -COO groups in 2,4-D and the $-\mathrm{OH}$ groups of LDHs were formed, and the bonding distances were 1.684 and $1.744 \AA$, respectively. The common range of the hydrogen bond distance is below $3.5 \AA .{ }^{38}$ According to the results, the maximum bonding distance is shorter than $3.5 \AA$, illustrating the stronger hydrogen bond interactions between LDHs and 2,4-D. Furthermore, these results can prove that the physisorption and chemisorption are coexistent in the adsorption process of 2,4-D onto LDHs.

\subsection{Adsorption mechanism}

Based on the abovementioned experimental and theoretical DFT calculations, the adsorption mechanism was proposed as follows: (a) the adsorption process was first driven by the electrostatic attraction between the CMS@LDH composites and oppositely charged $2,4-\mathrm{D}$; moreover, the $\pi-\pi$ interaction between 2,4-D and CMS enhanced the affinity of the CMS@LDH composites towards 2,4-D; (b) $\mathrm{Cl}^{-}$existing on the surface or in the interlayer of LDHs could exchange with 2,4-D; and (c) the hydrogen bond was formed between LDHs and 2,4-D during the adsorption process.

\section{Conclusions}

In this study, three different core-shell CMS@LDH composites were synthesized by direct fabrication of the LDH nanoplatelets onto carbon microspheres and then used as adsorbents to remove 2,4-D from an aqueous solution. The adsorption capacities were examined with various operational parameters such as solution $\mathrm{pH}$, contact time, and temperature. It was found that the adsorption of 2,4-D onto the CMS@LDH composites decreased with the increasing $\mathrm{pH}$. The adsorption of 2,4-D onto CMS@LDH composites could reach an equilibrium rapidly within $100 \mathrm{~min}$. The adsorption isotherm data could be accurately described by the Langmuir model. The maximum adsorption capacity of CMS@MgAl-LDHs, CMS@NiAl-LDHs, and CMS@ZnAl-LDHs for 2,4-D was 287.50,
265.99, and $223.02 \mathrm{mg} \mathrm{g}^{-1}$, respectively, at $298 \mathrm{~K}$. The experiment results indicated that the adsorption process was endothermic and spontaneous. The theoretical DFT calculation analysis suggested that the $\mathrm{LDH}$ shell could interact with 2,4-D through ion-exchange and hydrogen bonding. In addition, the CMS core reacted with 2,4-D through strong $\pi-\pi$ interactions. In summary, the core-shell CMS@LDH composites can constitute a promising adsorbent that offers a rapid and effective adsorption capacity for the removal of 2,4-D in an aqueous solution.

\section{Conflicts of interest}

There are no conflicts of interest to declare.

\section{Acknowledgements}

This work was supported by the National Science Foundation of China (no. 21677087) and the National Science and Technology Major Project of China "Environmental Protection Technology Integration and Key Equipment for Tight Reservoir Development" (2016ZX05040-005).

\section{Notes and references}

1 A. Chaparadza and J. M. Hossenlopp, J. Colloid Interface Sci., 2011, 363, 92-97.

2 F. Zhang, Y. Song, S. Song, R. Zhang and W. Hou, ACS Appl. Mater. Interfaces, 2015, 7, 7251-7263.

3 K. Nejati, S. Davary and M. Saati, Appl. Surf. Sci., 2013, 280, 67-73.

4 V. O. Njoku, M. A. Islam, M. Asif and B. H. Hameed, J. Environ. Manage., 2015, 154, 138-144.

5 Y. Tang, S. Luo, Y. Teng, C. Liu, X. Xu, X. Zhang and L. Chen, J. Hazard. Mater., 2012, 241, 323-330.

6 H. El Harmoudi, L. El Gaini, E. Daoudi, M. Rhazi, Y. Boughaleb, M. A. El Mhammedi, A. Migalska-Zalas and M. Bakasse, Opt. Mater., 2014, 36, 1471-1477.

7 S. Deng, R. Ma, Q. Yu, J. Huang and G. Yu, J. Hazard. Mater., 2009, 165, 408-414.

8 Y. Li, B. Gao, T. Wu, D. Sun, X. Li, B. Wang and F. Lu, Water Res., 2009, 43, 3067-3075.

9 F. Yao, H. Hu, S. Xu, R. Huo, Z. Zhao, F. Zhang and F. Xu, ACS Appl. Mater. Interfaces, 2015, 7, 3882-3887.

10 R.-X. Wang, T. Wen, X.-L. Wu and A.-W. Xu, RSC Adv., 2014, 4, 21802-21809.

11 L. P. Cardoso and J. B. Valim, J. Phys. Chem. Solids, 2006, 67, 987-993.

12 A. Legrouri, M. Lakraimi, A. Barroug, A. De Roy and J. P. Besse, Water Res., 2005, 39, 3441-3448.

13 I. Pavlovic, C. Barriga, M. C. Hermosin, J. Cornejo and M. A. Ulibarri, Appl. Clay Sci., 2005, 30, 125-133.

14 J. A. Gursky, S. D. Blough, C. Luna, C. Gomez, A. N. Luevano and E. A. Gardner, J. Am. Chem. Soc., 2006, 128, 8376-8377.

15 M. Shao, F. Ning, J. Zhao, M. Wei, D. G. Evans and X. Duan, J. Am. Chem. Soc., 2012, 134, 1071-1077.

16 X. Sun and Y. Li, Angew. Chem., Int. Ed., 2004, 43, 597-601. 
17 P. Gunawan and R. Xu, Chem. Mater., 2009, 21, 781-783.

18 Y. Lu, Y. Zhao, L. Yu, L. Dong, C. Shi, M. J. Hu, Y. J. Xu, L. P. Wen and S. H. Yu, Adv. Mater., 2010, 22, 1407-1411.

19 J. Gong, T. Liu, X. Wang, X. Hu and L. Zhang, Environ. Sci. Technol., 2011, 45, 6181-6187.

20 J. B. Fei, Y. Cui, X. H. Yan, W. Qi, Y. Yang, K. W. Wang, Q. He and J. B. Li, Adv. Mater., 2008, 20, 452-456.

21 C. Chen, P. Wang, T.-T. Lim, L. Liu, S. Liu and R. Xu, J. Mater. Chem. A, 2013, 1, 3877-3880.

22 L.-g. Yan, K. Yang, R.-r. Shan, T. Yan, J. Wei, S.-j. Yu, H.-q. Yu and B. Du, J. Colloid Interface Sci., 2015, 448, 508-516.

23 C. Lei, X. Zhu, B. Zhu, C. Jiang, Y. Le and J. Yu, J. Hazard. Mater., 2017, 321, 801-811.

24 M. J. Hernandez-Moreno, M. A. Ulibarri, J. L. Rendon and C. J. Serna, Phys. Chem. Miner., 1985, 12, 34-38.

25 M. A. Aramendıa, V. Borau, C. Jiménez, J. M. Luque, J. M. Marinas, J. R. Ruiz and F. J. Urbano, Appl. Catal., A, 2001, 216, 257-265.

26 V. Chandra, J. Park, Y. Chun, J. W. Lee, I.-C. Hwang and K. S. Kim, ACS Nano, 2010, 4, 3979-3986.
27 Y. Zou, X. Wang, Y. Ai, Y.-H. Liu, J.-X. Li, Y. Ji and X. Wang, Environ. Sci. Technol., 2016, 50, 3658-3667.

28 J. S. Valente, F. Tzompantzi, J. Prince, J. G. H. Cortez and R. Gomez, Appl. Catal., B, 2009, 90, 330-338.

29 H. Hu, A. Yu, E. Kim, B. Zhao, M. E. Itkis, E. Bekyarova and R. C. Haddon, J. Phys. Chem. B, 2005, 109, 11520-11524.

30 T. Hibino, Appl. Clay Sci., 2011, 54, 83-89.

31 I. Langmuir, J. Am. Chem. Soc., 1916, 38, 2221-2295.

32 H. Freundlich, J. Phys. Chem., 1906, 57, 385-470.

33 B. Zhang, L. Luan, R. Gao, F. Li, Y. Li and T. Wu, Colloids Surf., A, 2017, 520, 399-408.

34 M. Raoov, S. Mohamad and M. R. Abas, J. Hazard. Mater., 2013, 263, 501-516.

35 X. F. Sun, B. B. Guo, L. He, P. F. Xia and S. G. Wang, AIChE J, 2016, 62, 2154-2162.

36 Z. Jin, X. Wang, Y. Sun, Y. Ai and X. Wang, Environ. Sci. Technol., 2015, 49, 9168-9175.

37 A. Ahmadi, M. Kamfiroozi, J. Beheshtian and N. L. Hadipour, Struct. Chem., 2011, 22, 1261-1265.

38 P. Padma Kumar, A. G. Kalinichev and R. J. Kirkpatrick, J. Phys. Chem. B, 2006, 110, 3841-3844. 